\title{
Um Sistema de Baixo Custo para Monitoramento do Consumo de Energia em Casas Inteligentes
}

\author{
Title: A Low-cost System for Energy Consumption Monitoring in Smart Homes
}

Felipe Nipo Ferreira

Escola Politécnica de Pernambuco

Universidade de Pernambuco

50.720-001 - Recife, Brasil

fpnipo@gmail.com

\author{
Carmelo José Albanez Bastos Filho \\ Escola Politécnica de Pernambuco \\ Universidade de Pernambuco \\ 50.720-001 - Recife, Brasil \\ carmelo.filho@upe.br
}

\begin{abstract}
Resumo Motivado pelo cenário atual de desperdício de energia devido à falta de controle no consumo na sociedade, este trabalho propõe um sistema para monitoramento do consumo de eletrodomésticos em casas inteligentes, usando um medidor não invasivo, de baixo custo e fácil instalação. O medidor proposto utiliza o sensor de corrente SCT-013-030, conectado a uma placa Arduino capaz de enviar os dados de consumo via serial. Os testes com o medidor focaram na possibilidade de desagregar o consumo de cada eletrodoméstico, classificando-os através da análise do consumo agregado da casa. Os resultados mostraram que, considerando eletrodomésticos comuns e de alta potência como um refrigerador, um ar-condicionado e um micro-ondas, foi possivel extrair padrões dos dados de consumo de cada aparelho, tornando possivel a classificação desses eletrodomésticos. O medidor construído pode servir de base para trabalhos futuros que se proponham ao desenvolvimento de sistemas não invasivos de monitoramento de cargas que utilizem técnicas de inteligência computacional para classificação dos eletrodomésticos a partir de seus padrões de consumo.
\end{abstract}

Palavras-Chave: Energy disaggregation, Non-intrusive load monitoring, NILM, NIALM

\begin{abstract}
Driven by the current energy waste scenario due to the lack of energy consumption control in our society, this paper proposes an application for consumption monitoring system for smart homes, using a noninvasive, low-cost, and easy to install energy meter. The proposed meter uses the SCT-013-030 current sensor, connected to an Arduino board capable of sending the consumption data through serial communication. The system assessment is focused on the possibility to infer the appliances uses based on the entire house consumption, classifying them based on the aggregated consumption analysis. The results show, considering conventional high-power devices, such as a refrigerator, an air-conditioner, and a microwave, it is possible to extract patterns on the consumption data for each appliance, allowing the classification of these devices. This system may be used in future researches for the development of non-invasive load monitoring systems based on computational intelligence techniques to classify appliances based on their consumption pattern.
\end{abstract}

Keywords: Energy disaggregation, Non-intrusive load monitoring, NILM, NIALM 


\section{Introdução}

O mundo todo tem discutido soluções para a crise energética, que passa pelo fato de que o mundo utiliza majoritariamente fontes de energia não renováveis, que vão se esgotar na natureza em algum momento [7]. Soma-se a isso o fato de que a demanda por energia tem aumentado seguindo o crescimento econômico e a ineficiência dos produtos utilizados [7]. Seguindo essa tendência em algum momento no futuro poderá haver um colapso na relação entre produção e demanda de energia, e isso pode acarretar em vários problemas desde grandes retrações econômicas, a guerras por recursos $[7,15]$.

A renovação do sistema energético é tida como uma das mais profundas transições da história da humanidade, comparadas até a descoberta do fogo e do combustível [14]. As discussões em relação a essa renovação passam pelo balanceamento do trilema energético [7] (Figura 1).

Esse trilema ilustra a necessidade de equilibrar três fatores para renovar o sistema energético: A segurança, que significa termos estruturas confiáveis, evitando desastres como o ocorrido em Fukushima, e também utilizar fontes que sejam capazes de atender a demanda mundial atual; A equidade, a energia tem que ser disponibilizada a toda população e por um preço acessível; E a sustentabilidade, que implica usar fontes renováveis seguras, com baixa emissão de carbono, e melhorar a eficiência no consumo de energia [7].

No que tange o consumo eficiente, hoje o nosso cenário é de desperdício de energia, desde sua produção, passando pelo uso de aparelhos ineficientes, até a falta de controle e educação no consumo da nossa sociedade, não só na indústria, mas também nas casas.

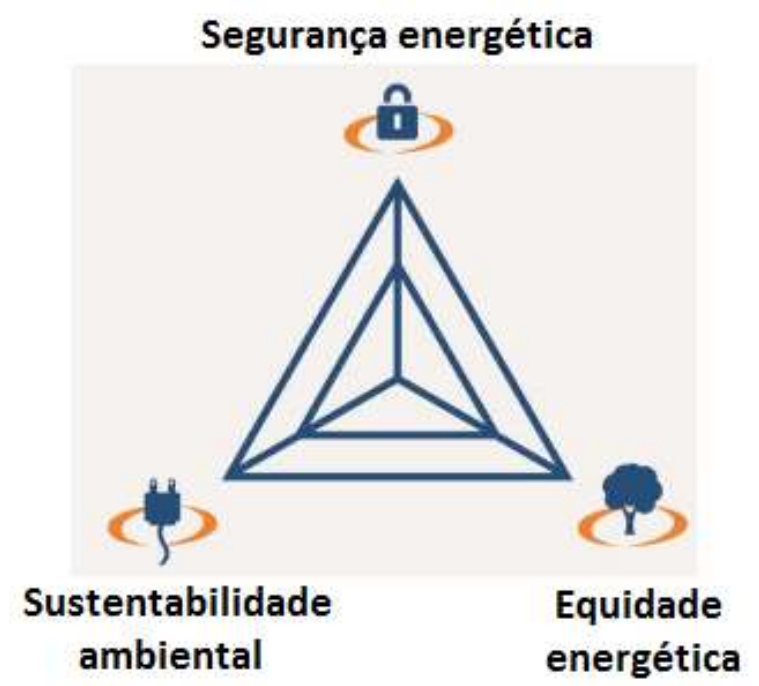

Figura 1: Trilema energético. Extraída de [7]
Ao contrário da crença comum de que a maior parte do consumo de energia na sociedade está relacionada às indústrias, pesquisas mostram que o comportamento cotidiano do ser humano, consumindo energia em casa ou em transporte, chega a $28 \%$ do total de energia consumida nos Estados Unidos, e a 26\% na União Europeia. Em ambientes comerciais, como escritórios, o consumo chega a $40 \%$ do consumo de energia total [2].

Apesar de haverem muitas pesquisas com foco na construção de eletrodomésticos de menor consumo, o processo de adoção dessas novas tecnologias pode ser longo e demorado, enquanto as novas casas podem já estar equipadas com esses novos eletrodomésticos, a maioria das casas ainda estará utilizando tecnologias antigas e ineficientes [2].

Assim soluções que visam aumentar a eficiência no consumo de energia com mínimas modificações nas casas podem ter um grande impacto no consumo global. Um problema fundamental para os consumidores domésticos é o de identificar o consumo de cada eletrodoméstico, com esses dados disponíveis seria possível para o consumidor monitorar e traçar um plano para redução do consumo em suas casas, munidos com dados não só em tempo real, mas em forma de histórico, tendo acesso ao consumo também em forma de preço através do cruzamento desses dados com os dados de tarifas da operadora de energia. O consumidor poderia ter acesso a sua conta de energia de uma forma similar às contas de telefones, com valores por eletrodomésticos.

O monitoramento do consumo de energia também tem sido uma abordagem promissora para solucionar um dos principais desafios e tema de pesquisas na área de computação ubíqua, que busca mapear o comportamento humano dentro de uma casa com abordagens de baixo custo e de fácil instalação. Isto porque o uso dos eletrodomésticos revela o que as pessoas estão fazendo em uma casa, permitindo essa abordagem também ser útil para construção de modelos preditivos [2].

Além disso, o monitoramento do consumo também pode ser útil para detecção precoce de falhas, alertando o usuário sobre possíveis avarias ou questões de segurança, como, por exemplo, a detecção de arcos elétricos que oferecem um potencial perigo de incêndio [2].

Este trabalho propõe um medidor não intrusivo de baixo custo e mínima instrumentação, utilizado para um sistema de monitoramento não intrusivo de cargas, para identificação dos eletrodomésticos ligados dentro de uma casa, através da extração de características de consumo desses aparelhos. Características espectrais como largura e amplitudes de picos, de vales, e a frequência entre eles, além dos valores de consumo, são utilizados para identificação de uma assinatura para vários eletrodomésticos. 


\section{Referencial Teórico}

\subsection{Conceitos}

Há duas abordagens para o monitoramento do consumo desagregado de uma casa, onde desagregado significa mapear o consumo por eletrodomésticos. A primeira é através de vários sensores distribuídos pela casa, e a segunda através de um único ponto de sensoriamento. Essa diferença básica entre essas duas abordagens impacta na complexidade do software, na facilidade de instalação, no custo do produto, e na precisão da medição [2].

Através da abordagem com sensores distribuídos, é possível obter uma maior precisão no consumo, porém requerendo um custo de instalação e manutenção muito elevado. Muitas vezes é necessário instalar um sensor para cada eletrodoméstico, onde cada um pode custar entre US\$ 30 e US\$ 100, sendo necessário ainda um dispositivo central sem fio para centralizar os dados, levando esses sistemas a um custo final da ordem de milhares de dólares. Em consequência da utilização de um sensor ou dispositivo por eletrodoméstico, essas abordagens adicionalmente oferecem a possibilidade de atuar e controlar esses eletrodomésticos, permitindo ligalos e desliga-los remotamente [2].

Sistemas de monitoramento não intrusivos de carga, ou NILM (Non Intrusive Load Monitoring), foram idealizados por Hart [5] e veem sendo estudados desde então com a proposta de utilizar um único ponto de sensoriamento buscando prover a mesma precisão de informação obtida através do sensoriamento distribuído, porém utilizando apenas um único e sofisticado sensor, explorando softwares complexos para inferir e desagregar o consumo de cada eletrodoméstico a partir do consumo total da casa $[2]$.
Hart [5] afirma que é possível classificar os eletrodomésticos em três tipos: Ligado/Desligado, que só possuem um estado de funcionamento enquanto ligados, como uma sanduicheira; Máquina de estados finita, que possuem vários estados de funcionamento, como uma máquina de lavar; E a Máquina de estados infinita, que varia o nível de consumo de acordo com alguma configuração de velocidade ou intensidade, como os amplificadores de áudio ou máquinas de costura. A Figura 2 ilustra os diferentes tipos de eletrodomésticos, com seus estados e transições de funcionamento: A Figura 2(A) representa um eletrodoméstico do tipo Ligado/Desligado, como uma sanduicheira; A Figura 2(B) ilustra uma máquina de estados finita de uma geladeira com função de descongelar; E a Figura 2(C) ilustra uma máquina de estados infinita de um amplificador, com vários estados de volumes.

A assinatura de um eletrodoméstico é a essência de sistemas de monitoramento não intrusivos de carga, essa assinatura pode ser definida como um conjunto de parâmetros que indicam a natureza ou estado de funcionamento de uma determinada carga [5].

Segundo Hart [5], essa assinatura pode ser gerada a partir de várias características elétricas extraídas a partir de diferentes objetos de análise, como mostra a Figura 3. Basicamente, considerando meios não intrusivos, é possível analisar o comportamento dos aparelhos pelos: Eventos de transição, ou seja, entre os estados de ligado e desligado, e vice-versa, além de transições entre os estados de funcionamento (Ver Figura 4); E pelo consumo regular.

No que diz respeito às transições, é possível extrair padrões analisando características como o formato, a amplitude, e a duração da onda, além da identificação de constantes de tempo. $\mathrm{Na}$ análise do consumo regular, é

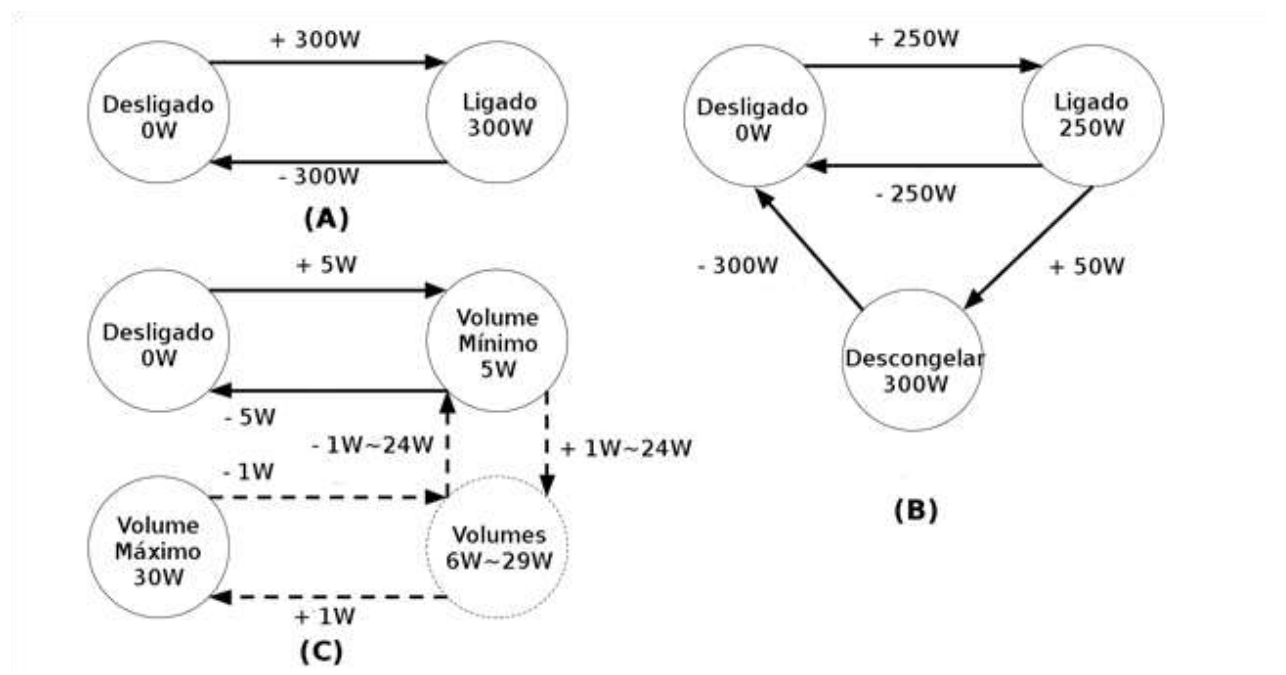

Figura 2: Tipos de eletrodomésticos. Extraída de [5] 
possível extrair padrões analisando características como: A frequência fundamental da potência, da corrente, e da admitância; A frequência da corrente dos harmônicos gerados pelos aparelhos, que é particularmente importante para identificar pequenos aparelhos, o qual é difícil de distinguir; E o consumo em corrente [5].

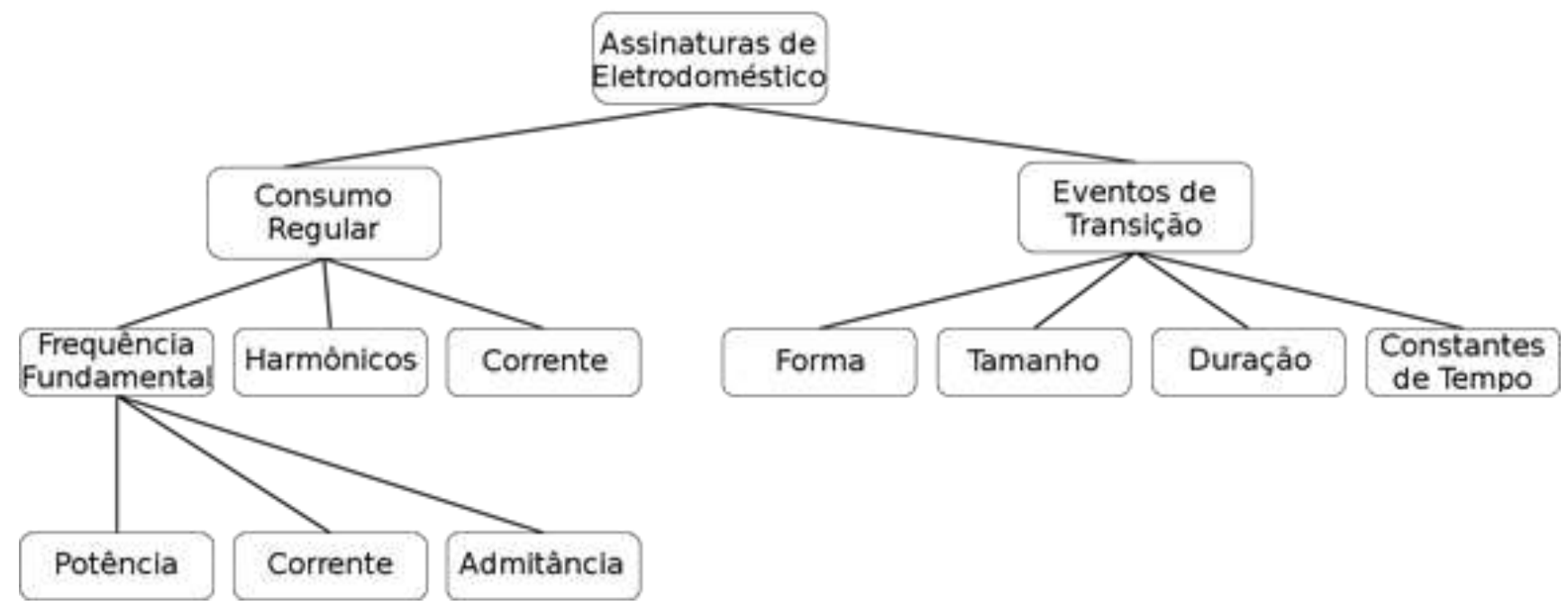

Figura 3: Parâmetros que formam a assinatura de um eletrodoméstico. Extraída de [5]

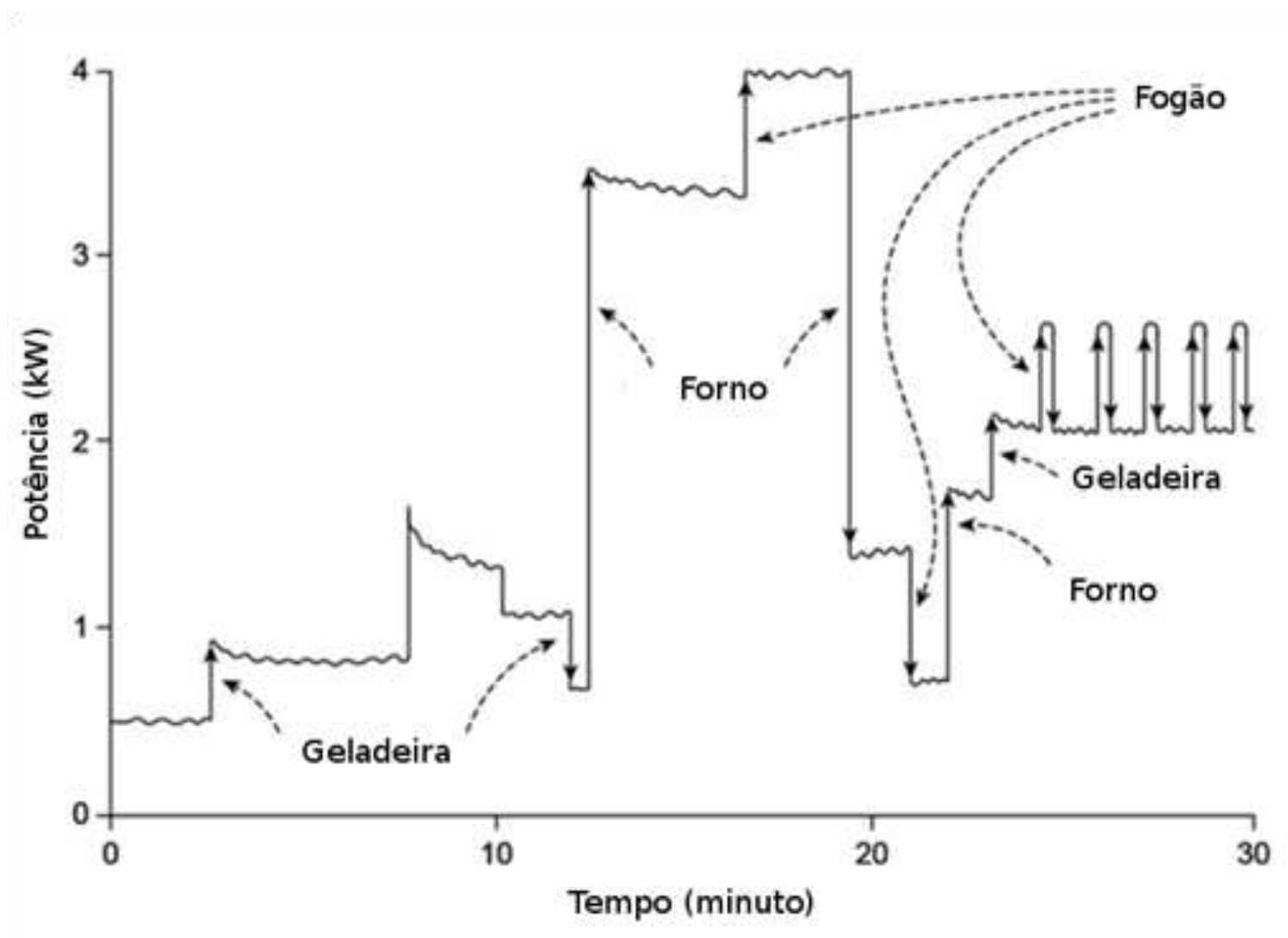

Figura 4: Eventos de transição. Extraída de [5] 
De acordo com Laughman, Kwangduk, Cox e Shaw [4], uma das vantagens da análise das transições é que essa técnica permite a identificação rápida quando um aparelho é ligado, porém o mesmo não é válido para quando um aparelho é desligado. Hart [5] cita esse problema em seu trabalho, afirmando que a maioria dos eletrodomésticos não possui um comportamento característico quando são desligados, dificultando a identificação desses por meio da análise das transições. Laughman, Kwangduk, Cox e Shaw [4], ainda levantam outro problema em relação à análise das transições, afirmando que essas podem ter menos sucesso em redes com muito ruído ou oscilações periódicas, mascarando os eventos de transições.

Ainda segundo Laughman e colaboradores [4], o comportamento da carga nos eventos de transição estão intimamente relacionados à sua construção física. As transições de um computador pessoal, são diferentes de uma lâmpada incandescente porque o comportamento energético nos capacitores de uma fonte de computador é fundamentalmente diferente do aquecimento do filamento de uma lâmpada, o que contribui para que os padrões sejam característicos para cada eletrodoméstico.

Além disso, segundo Leeb, Shaw e Kirtley [3], outra vantagem da análise de transições é que a maioria dos padrões de transição tende a ser preservados mesmo em aparelhos que usam moduladores de ondas ou corretores do fator de potência. Isso permite que esses sistemas sejam usados também em ambientes industriais e comerciais onde há muitos esforços desse tipo para estabilizar o consumo das cargas.

Laughman e colaboradores [4] concluem em seu trabalho que é possível obter uma melhor precisão em sistemas de monitoramento não intrusivos de carga, ao combinar a análise do consumo regular e dos eventos de transições.

\subsection{Trabalhos Relacionados}

Hart [5] construiu um sistema de monitoramento não intrusivo de cargas, utilizando um único sensor, baseado apenas na análise do consumo regular, considerando apenas eletrodomésticos do tipo Ligado/Desligado, e foi capaz de identificar os principais eletrodomésticos em quase todas as situações e casas testadas.

O trabalho de Nikolaev et al. [6], propõe uma abordagem baseada na análise dos eventos de transição, utilizando um único ponto de sensoriamento, usando essas informações para treinar uma rede neural. Os autores concluíram que o sistema foi capaz de reconhecer a maioria dos eletrodomésticos que representam a maior parcela da conta de luz, mesmo havendo a presença de distúrbios na rede, como variações de tensão e harmônicos.
O EletricSense é um sistema NILM, utilizando um único sensor, e baseia sua análise nas fortes interferências eletromagnéticas geradas ao ligar os eletrodomésticos. O EletricSense conseguiu uma precisão de $93,82 \%$ na detecção e identificação dos eletrodomésticos ligados [2].

Valchev, Marinov e Dimitrova [1] apresentam uma abordagem para medição de consumo de uma casa adicionando a cada eletrodoméstico um sensor não invasivo e que não necessita de alimentação, sendo capaz de gerar durante a medição energia suficiente para o sensoriamento e envio dos dados via rede sem fio para um dispositivo central.

\section{Metodologia}

Esta seção apresenta os procedimentos metodológicos utilizados para o desenvolvimento deste trabalho com o objetivo de construir um medidor não intrusivo para monitoração do consumo de uma casa. A construção desse medidor visou a futura transformação do mesmo em um produto, levando em consideração aspectos como custo e facilidade de instalação.

Em linhas gerais, esta pesquisa é de natureza aplicada, com uma abordagem quantitativa, objetivos descritivos e usando procedimentos experimentais. Esta monografia é uma pesquisa aplicada, pois, segundo Silva e Menezes [8], essa pesquisa gera conhecimentos práticos para solução de problemas específicos; Quantitativa, pois traduz as informações em números para classificação e análise; Descritiva, pois descreve características de determinado fenômeno e estabelece relações entre variáveis, usando técnicas padronizadas para coleta de dados; E Experimental, pois seleciona as variáveis que influenciam o objeto de estudo, e define as formas de controle e de observação dos efeitos que a variável produz no objeto. Em outras palavras, o ponto básico do procedimento experimental é a realização do experimento, que atua sobre o objeto de estudo, dentro de um ambiente controlado.

Como informação adicional para esta monografia, vale ressaltar que o levantamento bibliográfico para o referencial teórico foi realizado através de uma busca abrangente por títulos que abordam a redução do consumo de energia em casas inteligentes através de sistemas não intrusivos de monitoramento de cargas, incluindo artigos das principais bibliotecas digitais, como IEEE e ACM.

A organização desta monografia buscou a construção um medidor não intrusivo de baixo custo e fácil instalação, para depois apresentar e discutir os resultados obtidos sobre o consumo. Esse medidor foi construído utilizando apenas um único sensor que pode ser acoplado ao quadro de distribuição de energia para monitoração do 
consumo total de uma casa, ou sendo acoplado a uma tomada ou eletrodoméstico para análise isolada de uma carga. Os testes realizados visaram analisar a possibilidade de identificação de padrões e classificação dos eletrodomésticos baseando-se em dados de consumo coletados através do medidor construído neste trabalho.

\subsection{Medidor}

Foi utilizado um sensor de corrente alternada baseado no transformador de corrente SCT-013-030 fabricado pela YHDC, que é um sensor não invasivo capaz de transformar correntes de até $30 \mathrm{~A}$ em sinais de pequena amplitude, entre 0 e $50 \mathrm{mV}$, com uma margem de erro de $300 \mathrm{~mA}$ na medição. Esse sensor além de ser de baixo custo, em torno de US\$ 6, permite fácil instalação do sistema, sendo necessário apenas acoplar o sensor em torno do fio. Esse sensor serve de base em sistemas de monitoramento de energia como o Open Energy Monitor [11].

Para leitura do sensor no Arduíno foi preciso construir um circuito, apresentado na Figura 5, com a conexão do sensor ao Arduíno ilustrado na Figura 6. Esse circuito utiliza um resistor de carga de $22 \Omega$ para o sensor, devido a esse não possuir resistor de carga interno. Além disso, o circuito utiliza polarização de corrente contínua, adicionando um ganho de $2,5 \mathrm{~V}$ para adequar a saída do circuito a entrada analógica do Arduíno, para isso utilizando um divisor de tensão com dois resistores de $10 \mathrm{k} \Omega$ e um capacitor de $10 \mathrm{uF}$.

Foi utilizada uma placa Arduíno UNO R3 como unidade de processamento dos dados advindos do sensor e funcionando como interface de comunicação para obter os dados e apresentar com gráficos de consumo em um software para PC. O Arduíno é uma plataforma adequada para prototipação rápida e provas de conceito, baseada no micro controlador ATMEL ATMEGA16U2, operando a $16 \mathrm{MHz}$, e contando com conversores analógicos digitais, ou ADC, utilizado neste projeto. Além disso, essa placa contém conversor USB-serial, com saída USB permitindo comunicação serial com um PC [9].

O firmware construído para Arduíno contempla a configuração e leitura do sensor através do $\mathrm{ADC} 1$ pelo pino A1, além da configuração da comunicação serial para envio dos dados ao software para o PC a cada 500 milissegundos, gerando uma taxa de amostragem de 2 amostras/segundo. O firmware utiliza a biblioteca EmonLib [12] para interação com o sensor, e foi construído baseando-se no estudo do funcionamento do firmware "emonTxV3_DirectSerial" do OpenEnergyMonitor [13]. Os dados de consumo são enviados via comunicação serial a um software em PC.

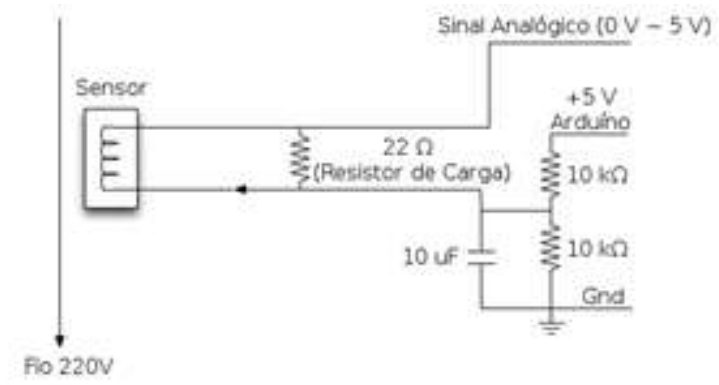

Figura 5: Circuito para conexão do sensor ao Arduíno.

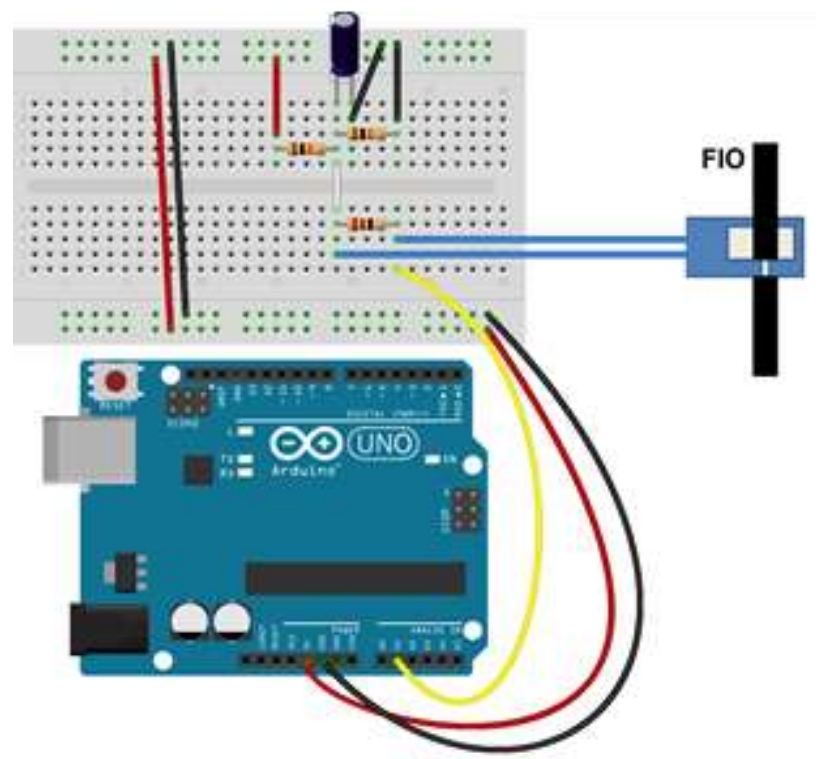

Figura 6: Conexão do sensor ao Arduíno.

O Emon [10], software local para PC construído durante este trabalho, foi utilizado para apresentação dos dados de consumo em forma de gráficos permitindo sua análise, sendo útil neste trabalho para identificação de padrões de consumo. Esse software foi desenvolvido usando NodeJS, versão 4.4.5, que é uma plataforma que permite o reuso de código para criação de software para diversos ambientes como: Localmente, seja em Windows, Linux, ou MacOS; Na internet; E também em dispositivos móveis, como Android e iOS. Sendo interessante para um futuro produto onde o usuário possa monitorar seu consumo tanto localmente, quanto remotamente pela internet, ou smartphone. Foi utilizado o NodeWebkit, versão 0.12 .3 , que permite a execução do código NodeJS como uma aplicação local, e a biblioteca Chart.JS, versão 2.1.4, para exibição de gráficos no software, e a biblioteca FFT-JS, versão 0.0.9, para cálculo da FFT, sendo baseado no algoritmo de Cooley-Tukey Radix-2, possibilitando a apresentação dos dados de consumo no domínio do tempo. Além disso, foi utilizada a biblioteca Bootstrap, versão 3.3.6, para construção da interface gráfica do sistema. 


\subsection{Validação experimental}

Para os testes foram selecionados eletrodomésticos comuns, que estão presentes na maioria das casas na cidade de Recife, de alta potência, e que representam uns dos maiores vilões de consumo nas casas. Os eletrodomésticos utilizados foram: Um refrigerador, modelo com freezer e capacidade para 480 litros da marca Consul, modelo "Biplex 480"; Um micro-ondas, com capacidade de 19 litros da marca Panasonic, modelo "Dia-a-dia" NNST254W; E um Ar-condicionado, modelo de janela com capacidade de 10.000 BTUs da marca Consul, modelo CCN10DB.

Os eletrodomésticos foram monitorados isoladamente, com o medidor sendo acoplado diretamente ao fio do eletrodoméstico, onde esses foram ligados e desligados repetidamente para identificação de padrões durante eventos de transição. Os dados de consumo foram gravados em arquivo para posterior análise, onde foram coletadas 10 amostras de eventos de transição, para cada tipo de transição de cada aparelho, com o intuito de extrair métricas que são apresentadas na seção de resultados desta monografia. $\mathrm{O}$ número de amostras coletadas foi definido empiricamente, utilizando como base a baixa variação de comportamento das ondas em cada amostra.

Além disso, foram coletadas 512 amostras sequenciais em uma medição única desses eletrodomésticos durante pleno funcionamento, sem a ocorrência de eventos de transição, com o objetivo de analisar o consumo regular desses aparelhos

\section{Resultados}

Nesta seção são apresentados os resultados obtidos a partir dos testes realizados com o medidor construído nesta monografia, onde todas as figuras com gráficos de consumo de energia apresentados foram extraídas a partir da utilização do software Emon, onde as informações de consumo nos gráficos estão na unidade de Watts.

As Figuras 7 e 8 apresentam os gráficos de consumo do refrigerador selecionado como objeto de teste neste trabalho. A parte do gráfico circulada na Figura 7 ilustra o evento de transição quando o refrigerador foi ligado, onde ocorre um pico de em média 1238,63 watts, com um desvio padrão de 91,31 watts, que se mantém por entre 0,5 e 3,5 segundos. Após esse pico inicial o consumo cai para em média 129,77 watts, com um desvio padrão de 13,52 watts. O gráfico na Figura 8 apresenta o consumo regular do refrigerador, que é de em média 86,52 watts, com desvio padrão de 1,78 watts.

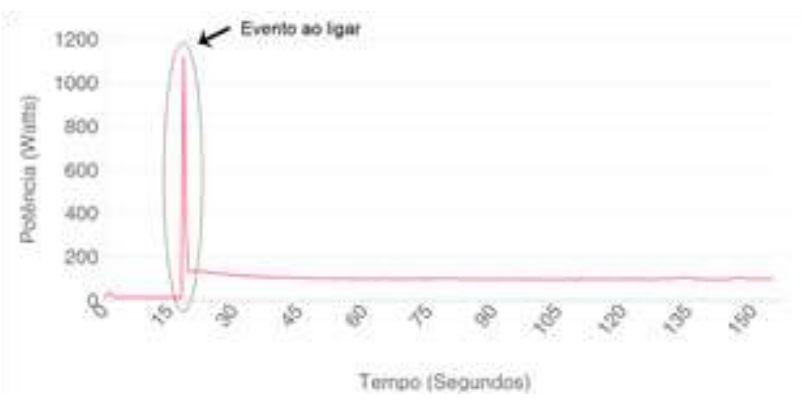

Figura 7: Evento de transição no consumo ao ligar o refrigerador

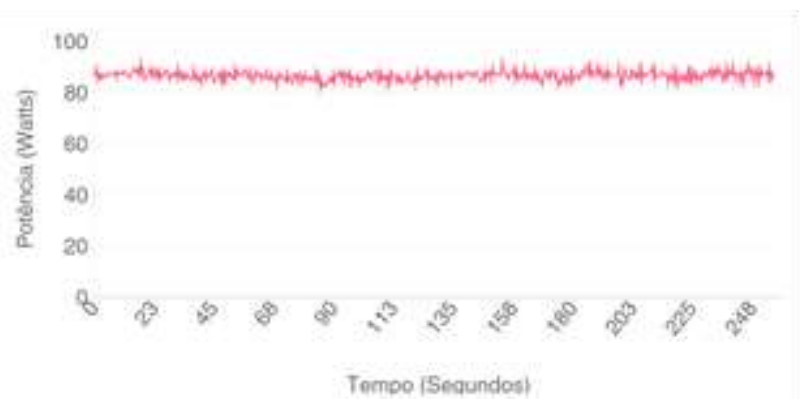

Figura 8: Consumo regular do refrigerador

As Figuras 9 e 10 apresentam os gráficos de consumo do micro-ondas selecionado como objeto de teste neste trabalho. A área circulada no gráfico da Figura 9 representa o evento de transição quando o micro-ondas foi ligado, onde ocorre um primeiro pico de em média 417,79 watts, podendo variar bastante com um desvio padrão de 94,11 watts. Em seguida o consumo diminui formando um vale, caindo para em média 321,99 watts, com um desvio padrão de 4,49 watts, quando ocorre um segundo pico maior, elevando o consumo para em média 575,33 watts, com um desvio padrão de 24,46 watts. O gráfico na Figura 10 permite analisar o consumo regular do micro-ondas, que é de em média 520,34 watts, com um desvio padrão de 10,62 watts.

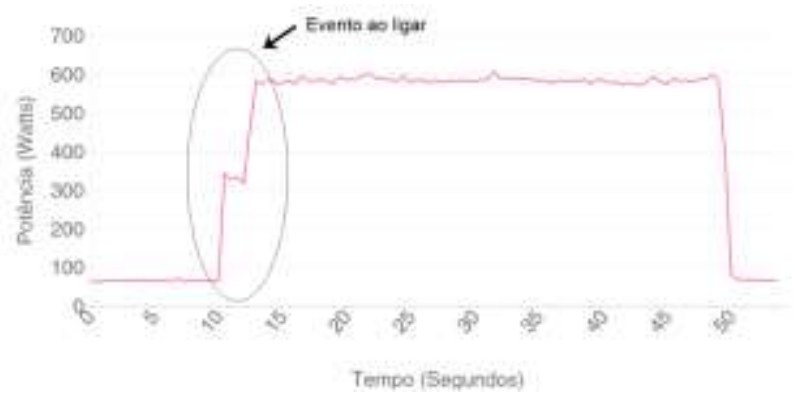

Figura 9: Evento de transição no consumo ao ligar o micro-ondas. 


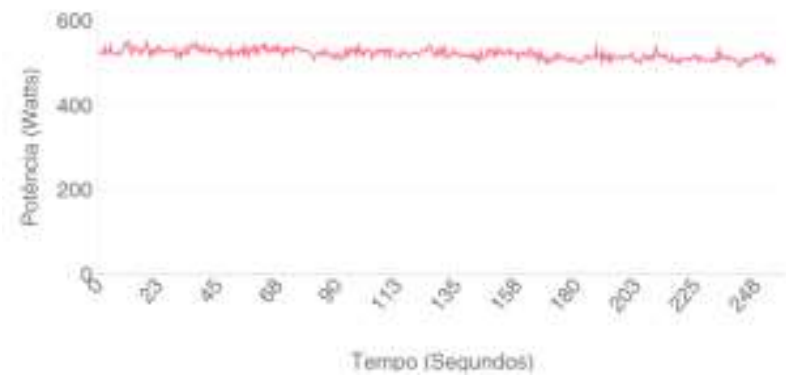

Figura 10: Consumo regular do micro-ondas.

Os gráficos de consumo do ar-condicionado selecionado como objeto de teste neste trabalho são apresentados nas Figuras 11 e 12. A Figura 11 ilustra dois eventos de transição identificados durante as medições do arcondicionado, o primeiro, que corresponde à área circulada no gráfico, é referente ao evento de transição no consumo quando o aparelho é ligado, onde ocorre um pico de em média 79,05 watts, com um desvio padrão de 1,79 watts, seguido de um consumo regular de em média 70,8 watts, com um desvio padrão de 3.26 watts, se mantendo nesse nível por entre 11 e 38 segundos. Após isso ocorre um segundo evento de transição, que é ilustrado na área contornada por um quadrado no gráfico, e é referente ao momento quando o compressor interno do arcondicionado é ligado de fato, onde ocorre um pico de em média 1280,21 watts, com um desvio padrão de 118,07 watts, seguido de uma queda no consumo formando um vale de em média 280,98 watts, com um desvio padrão de 18,7 watts. O gráfico na Figura 12 apresenta o consumo regular do ar-condicionado, que é de em média 369,79 watts, com um desvio padrão de 3,8 watts.

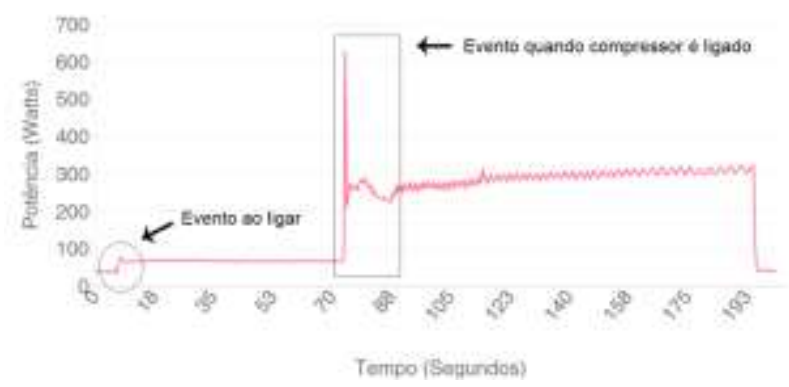

Figura 11: Eventos de transição no consumo do ar-condicionado.

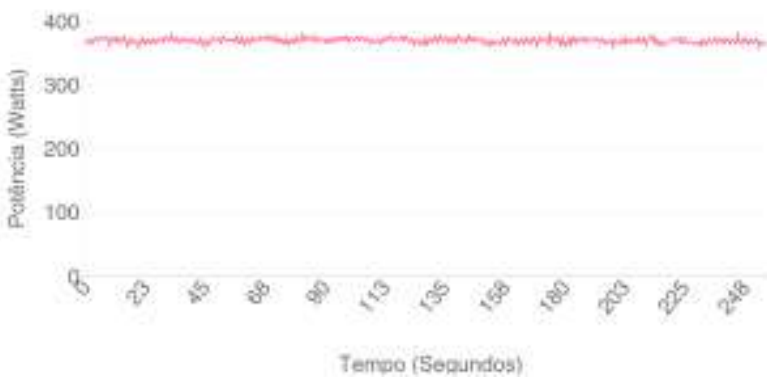

Figura 12: Consumo regular do ar-condicionado com seu compressor interno ligado.

\section{Conclusão}

Este trabalho foi motivado pelo cenário, visto atualmente, de desperdício de energia, em partes devido à falta de controle e educação no consumo na nossa sociedade, onde grande parte do consumo se concentra nas casas, transportes e ambientes comerciais. Devido ao lento processo de adoção, por parte das casas, de novos eletrodomésticos otimizados para consumo, soluções que visam aumentar a eficiência no consumo de energia nas casas de forma fácil, exigindo mínimas modificações, podem ter um grande impacto no consumo global.

Dessa forma, o sistema construído nesta monografia se propõe a monitorar o consumo de energia de uma casa, através de um medidor não invasivo, de baixo custo e fácil instalação. Além disso, esta monografia também propõe esse medidor como base para sistemas não intrusivos de monitoramento de cargas baseado em inteligência computacional.

Para construção do medidor foi utilizado o sensor de corrente SCT-013-030, conectado a uma placa Arduíno que permite o envio dos dados de consumo via comunicação serial para um software de análise em computador.

Os testes foram realizados monitorando os aparelhos isoladamente, sendo eles um refrigerador, um micro ondas, e um ar-condicionado, que são eletrodomésticos de alta potência, e representam uns dos maiores vilões de consumo nas casas. Para cada tipo de evento de transição em cada aparelho foram realizadas repetidas medições, para extração de métricas que tornem possível a classificação de cada eletrodoméstico de acordo com seu consumo.

Os resultados obtidos com o medidor mostram que os dados coletados foram suficientes para detectar métricas que caracterizam os eletrodomésticos, levando em consideração a análise de padrões nas ondas de consumo em eventos de transição ao ligar, analisando características como o formato, amplitude e duração de tempo. O refrigerador selecionado, ao ser ligado, tende a apresentar sempre um grande pico, em torno de 1239 watts, seguido 
queda no consumo para em torno de 130 watts, e em seguida estabilizando em torno de 86 watts. O microondas selecionado, quando é ligado, tende a apresentar inicialmente um pico bastante variável, de em média 418 watts, reduzindo o consumo logo após formando um vale de em torno de 322 watts, seguido de um segundo e maior pico de em torno de 575 watts, e por fim estabilizando o consumo em torno de 520 watts. Por fim, o arcondicionado selecionado, quando tem seu compressor interno ligado, apresenta um grande pico em torno de 1280 watts, seguido de um vale de em torno de 281 watts, e por fim estabilizando o consumo em torno de 370 watts.

Através dos resultados obtidos é possível observar que, nos cenários propostos, os padrões das ondas apresentam diferenças para cada eletrodoméstico testado, sendo analisados tanto no domínio do tempo como da frequência, considerando momentos de transição e também de consumo regular, sendo suficientemente distintos para a extração de métricas para identificação desses aparelhos, tornando possível a classificação de eletrodomésticos utilizando o medidor construído nesta monografia.

Portanto, o medidor de energia construído neste trabalho pode servir de base para trabalhos futuros que se proponham ao desenvolvimento de sistemas não invasivos de monitoramento de cargas que utilize de técnicas de inteligência computacional, como as redes neurais artificiais, para inferir aparelhos ligados dentro de uma casa, ambientes comerciais, ou indústrias, tomando como base o consumo geral ou de partes do ambiente. Esses sistemas podem ainda se basear em informações contextuais, aprendendo a rotina da casa e seus moradores para aumentar sua precisão, podendo também atuar como um sistema de automação residencial, ligando e desligando eletrodomésticos de acordo com a rotina aprendida. Por fim, essas informações de consumo desagregadas por eletrodomésticos podem ser úteis ao usuário para gerenciamento de seu consumo de energia, permitindo que o mesmo trace estratégias para redução de consumo, e também possibilitando o monitoramento remoto e em tempo real dos aparelhos que estão sendo utilizados, servindo como entrada para sistemas que se proponham a mapear as atividades de pessoas dentro de uma casa.

Considerando uma futura transformação em produto do medidor proposto neste trabalho, pode ser interessante adicionar ao dispositivo a capacidade de comunicação via rede sem fio, havendo um dispositivo central para receber todos os dados e processá-los. O dispositivo pode ser acoplado ao quadro geral de energia de uma residência, ou acoplado por eletrodomésticos, até mesmo em forma de adaptador de tomadas.

\section{Referências}

[1] V. C. Valchev, A. St. Marinov, E. N. Dimitrova. Self Powered Current Acquisition System with Wireless Data Transfer. Information \& Communication Technology Electronics \& Microelectronics (MIPRO). páginas 108-112, 2013.

[2] S. Gupta, M. S. Reynolds, S. N. Patel. ElectriSense: Single-point Sensing Using EMI for Electrical Event Detection and Classification in the Home. Proceedings of the 12th ACM International Conference on Ubiquitous Computing (UbiComp '10). páginas 139-148, 2010.

[3] S. B. Leeb, S. R. Shaw, J. L. Kirtley. Transient Event Detection in Spectral Envelope Estimates for Nonintrusive Load Monitoring. IEEE Transactions on Power Delivery. 10(3):1200-1210, 2002.

[4] C. Laughman, K. Lee, R. Cox, S. Shaw. Power Signature Analysis. IEEE Power and Energy Magazine. 1(2):56-63, 2003.

[5] G. W. Hart. Nonintrusive Appliance Load Monitoring. Proceedings of the IEEE. 80(12):18701891, 2002.

[6] N. Nikolaev, Y. Rangelov, V. Valchev, A. Marinov. Technique for Indirect Analysis of Domestic Power Consumers based on Power Pattern Recognition for Smart Energy Metering. Information \& Communication Technology Electronics \& Microelectronics (MIPRO). páginas 971974, 2013.

[7] World Energy Council. 2014 World Energy Issues Monitor. http://www.worldenergy.org/wpcontent/uploads/2014/01/World-Energy-IssuesMonitor-2014.pdf, Set. 2016.

[8] E. L. Silva, E. M. Menezes. Metodologia da pesquisa e elaboração de dissertação. Laboratório de Ensino a Distância da UFSC, Florianópolis, 2001.

[9] Arduino. Arduino/Genuino UNO. https://www.arduino.cc/en/Main/ArduinoBoard Uno, Ago. 2016.

$[10]$

Github. energy-monitor. https://github.com/fnipo/energy-monitor, Ago. 2016.

[11] OpenEnergyMonitor. emonTx V3. https://openenergymonitor.org/emon/modules/e monTxV3, Ago. 2016. 
[12] Github. EmonLib. https://github.com/openenergymonitor/EmonLib, Ago. 2016.

[13] Github. emonTxV3_DirectSerial. https://github.com/openenergymonitor/emonTxF irmware/tree/master/emonTxV3/noRF/emonTxV3 DirectSerial, Ago. 2016.

[14] A. Lovins. A 40-year plan for energy. https://www.ted.com/talks/amory_lovins_a 50 year_plan for_energy, Set. 2016.

[15] V. L. Giorgia. Oil wars, energy crisis, and Thomas Edison's solution. http://www.inquisitr.com/3143779/oil-warsenergy-crisis-and-thomas-edisons-solution, Set. 2016. 